Provided for non-commercial research and education use. Not for reproduction, distribution or commercial use.

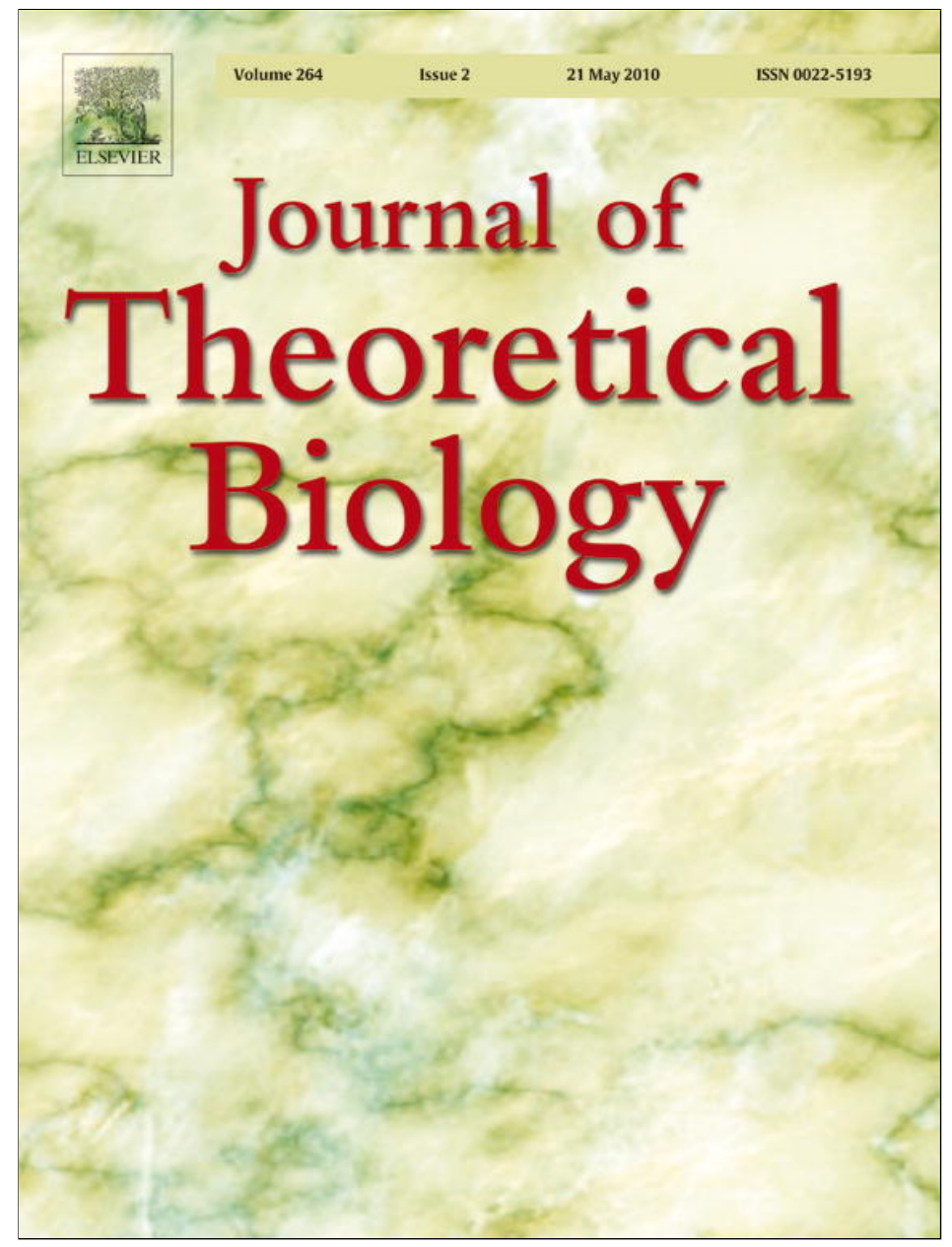

This article appeared in a journal published by Elsevier. The attached copy is furnished to the author for internal non-commercial research and education use, including for instruction at the authors institution and sharing with colleagues.

Other uses, including reproduction and distribution, or selling or licensing copies, or posting to personal, institutional or third party websites are prohibited.

In most cases authors are permitted to post their version of the article (e.g. in Word or Tex form) to their personal website or institutional repository. Authors requiring further information regarding Elsevier's archiving and manuscript policies are encouraged to visit:

http://www.elsevier.com/copyright 


\title{
Regulatory dynamics of standard two-component systems in bacteria
}

\author{
Beatriz Carely Luna Olivera ${ }^{\mathrm{a}}$, Edgardo Ugalde ${ }^{\mathrm{a}, *}$, Agustino Martínez-Antonio $^{\mathrm{b}, * *}$ \\ a Instituto de Física, Universidad Autónoma de San Luis Potosí, 78000 San Luis Potosí, Mexico \\ bepartamento de Ingeniería Genética, Centro de Investigación y de Estudios Avanzados del Instituto Politécnico Nacional, Irapuato 36500, Mexico
}

\section{A R T I C L E I N F O}

Article history:

Received 19 March 2009

Received in revised form

5 February 2010

Accepted 5 February 2010

Available online 26 February 2010

Keywords:

Dynamics modeling

Environmental sensing

Synthetic biology

\begin{abstract}
A B S T R A C T
Complex cellular networks regulate metabolism, environmental adaptation, and phenotypic changes in biological systems. Among the elements forming regulatory networks in bacteria are regulatory proteins such as transcription factors, which respond to exogenous and endogenous conditions. To perceive their surroundings, bacteria have evolved sensory regulatory systems of two-components. The archetype of these systems is made up of two proteins-a signal sensor and a response regulator-whose genes are usually located together in a single transcription unit. These units switch transcriptional programs in response to environmental conditions. Here, we study 14 two-component systems in Escherichia coli, which have been experimentally characterized with respect to their transcriptional regulation and their perceived signal. Given that the activity of these sensory units is connected to the rest of the transcriptional network, we first classify them as autonomous, semiautonomous or dependent, according to whether or not they use additional regulators to be transcribed. Next, we use discrete-time models to simulate their qualitative regulatory dynamics in response to their transcriptional regulation and to the activation of these systems by their cognate signals. Compared to more traditional ordinary differential equations method, ours has the advantage of being computationally simple and mathematically tractable, while keeping the ability to reproduce the phenomenology described by non-linear models. The aim of the present work is not the study of all possible behaviors of these two-component systems, but to exemplify those behaviors reported in the literature. On the other hand, most of these systems are auto-activating switches, a property that distinguishes them from the other transcription factors in the regulatory network, which are mostly auto-repressing. Based on the data, our models show dynamic behaviors that explain how most of these sensory systems convey abilities for multistationarity, and these dynamic properties could explain the phenotypic heterogeneity observed in bacterial populations. Our results are likely to have an impact in the design of synthetic signaling modules.
\end{abstract}

(c) 2010 Elsevier Ltd. All rights reserved.

\section{Introduction}

For the purpose of changing transcriptional programs in response to environmental conditions, bacteria use simple sensory systems of two-components (Gao and Stock, 2009; Igo et al., 1990; Mascher et al., 2006). The particularity of these systems is that they perceive exogenous signals (mostly related to conditions of physicochemical stress) and communicate this information to the cell through a signal transduction process by means of a phosphotransfer reaction from the sensory to the regulatory component. This distinguishes two-component systems from the so-called one-component systems where the TFs directly bind their specific signal effectors (mostly small-molecule metabolites).

\footnotetext{
Abbreviation: TFs, transcription factors

* Corresponding author.

** Corresponding author. Tel.: +52462 6239673 .

E-mail addresses: ugalde@ifisica.uaslp.mx (E. Ugalde), amartinez@ira.cinvestav.mx (A. Martínez-Antonio).
}

In Escherichia coli, 27 of the 30 pairs of two-component systems genes are located in single transcription units (Martinez-Antonio et al., 2006). Sensory proteins normally contain several transmembrane folds responsible for periplasm anchoring. Their N-terminal domain senses environmental signals leading to conformational changes that result in autophosphorylation of a conserved histidine residue (a mostly ATP dependant reaction). This phosphate group is then transferred to an aspartate residue of the response-regulatory protein. This transphosphorylation process might be facilitated through protein-protein interactions between the sensor and regulatory proteins as observed in several two-component systems (Guhaniyogi et al., 2008; Whitworth et al., 2008; Workentine et al., 2009; Zhao et al., 2008). Following phosphorylation, the regulatory protein-which is itself a transcription factor (TF) - will diffuse into the cytoplasm and reach its DNA-binding sites to regulate the expression of relevant genes.

$E$. coli is the best characterized bacterial model with respect to the function of molecular components, including those constituting their transcriptional regulatory network. Due to their regulatory inputs at 
the end of longest regulatory cascades two-component systems probably defines adaptive or development processes in this bacterium (Martinez-Antonio et al., 2008). Nevertheless, the dynamics of these sensory systems - as a part of the whole regulatory network - is largely unknown. In this work, using discrete-time mathematical models, we initiate a qualitative study of the dynamics of the most classical two-component systems in E. coli. For this purpose, we first classify the two-component systems, considering whether they require additional regulators to be transcribed. We then propose a simple graphical representation of these systems and analyze their dynamic behavior. In our analyses we take into account the different kinds of transcriptional input regulating the behavior of the system, and we distinguish four possible dynamical responses: the resting state, the induced state, the turn off and multistationarity (defined below). It is important to emphasize that in this study we do not pretend to model all the particular dynamics of two-component systems in bacteria (as many particular properties are as yet unknown) but we initiate the study of those properties that could be common to all of them. We further discuss some plausible implications for $E$. coli physiology of the different qualitative dynamics analyzed and their possible impact in the design and construction of signal-sensing transcriptional circuits.

\section{Results}

\subsection{Sub-classification of two-component systems based on their autonomous activity}

The genome of $E$. coli reveals the existence of around 30 two-component systems (Blattner et al., 1997; Oshima et al., 2002). Of these, 21 are annotated in Ecocyc (Keseler et al., 2005), and a subset of 14 also has additional information on their environmental effectors (Table 1), (Martinez-Antonio et al., 2006; Salgado et al., 2006). In this work, we further analyze this subset of 14 two-component systems. The sensory and regulatory genes of all these 14 two-component systems are encoded in single transcription units (Fig. 1a), and their sensor proteins are either predicted or known to be localized in the periplasm (with the exception of GlnLG, in which the sensor protein is located in the cytoplasm). Given that perception of signals from the environment is the common task of these systems, a simple classification can be based on whether they show auto-regulation of their own transcription or require additional TFs (Fig. 1b). Such a classification gives information on the coordination between these environment-sensing transcriptional modules and the rest of the transcriptional network of this bacterium (Fig. 1c). According to alternative modes of regulation for gene expression, two-component systems can be grouped in: (i) autonomous (systems with autogenous regulation); (ii) semiautonomous (systems with both autogenous regulation and regulation by other transcription factors); and (iii) dependent (systems only regulated by other TFs). Remarkably, 8 of the 10 auto-regulated systems are positively autoregulated, while only two possess another type of auto-regulation, which will be widely explained in another section of this work. The lack of solely auto-repressed systems contrasts with many other TFs in E. coli, where negative auto-regulation is observed in around $60 \%$ of the cases (Martinez-Antonio et al., 2008). This means that the nature of these two-component sensory systems is similar to positive switches, a recurrent kind of feedback loop found in the core of all differentiation processes giving rise to "memory" or "inertial effects" (see below), (Guespin-Michel and Kaufman, 2001; Thomas and D'Ari, 1990). In contrast, TFs with negative auto-regulation seem to be inherent to regulatory feedback loops maintaining homeostasis in other areas of metabolism (not analyzed here).

\subsection{Graphical representation of the two-component sensory systems}

Biological networks have been formally represented as nodes and arrows, where nodes represent molecular entities (e.g. proteins) and arrows their functional interactions (Glass and Kauffman, 1973; Thomas, 1973). In this way, in a transcriptional regulatory network,

Table 1

Sub-classification of two-component systems on the basis of their autonomous transcription.

\begin{tabular}{|c|c|c|c|c|c|}
\hline $\begin{array}{l}\text { Two-component } \\
\text { systems }\end{array}$ & Cognate signal & $\begin{array}{l}\text { Mode of } \\
\text { regulation } \\
\text { of target } \\
\text { genes }\end{array}$ & $\begin{array}{l}\text { Mode of autogenous } \\
\text { regulation } \\
\text { (auto-regulation) }\end{array}$ & $\begin{array}{l}\text { Regulated by other TFs } \\
\text { (mode) }\end{array}$ & References (experimental studies) \\
\hline \multicolumn{6}{|c|}{ Autonomous systems } \\
\hline CpxRA & $\begin{array}{l}\text { Misfolded membrane } \\
\text { proteins. }\end{array}$ & Dual & Positive & No & DiGiuseppe and Silhavy (2003), Otto and Silhavy (2002) \\
\hline CusSR & Copper resistance & Positive & Positive & No & Munson et al. (2000), Yamamoto and Ishihama (2005) \\
\hline EvgAS & Multidrug resistance & Positive & Positive & No & Nishino et al. (2005), Tanabe et al. (1998) \\
\hline QseBC & Autoinducer-2 & Positive & Positive & No & Sperandio et al. (2002) \\
\hline PhoBR & Inorganic phosphate & Positive & Positive & No & Wanner and Chang (1987) \\
\hline KdpDE & Potassium ions & Positive & Positive & No & $\begin{array}{l}\text { Asha and Gowrishankar (1993), Walderhaug et al. } \\
\text { (1992) }\end{array}$ \\
\hline PhoPQ & Magnesium ions & Dual & $\begin{array}{l}\text { Dual (positive and } \\
\text { negative) }\end{array}$ & No & Kasahara et al. (1992), Kato et al. (1999) \\
\hline \multicolumn{6}{|c|}{ Semiautonomous systems } \\
\hline BaeSR & Tannins, indoles & Positive & Positive & CpxRA (positive) & $\begin{array}{l}\text { Hirakawa et al. (2005), Leonhartsberger et al. (2001), } \\
\text { Zoetendal et al. (2008) }\end{array}$ \\
\hline ZraSR & Zinc, lead & Positive & Positive & CRP (positive) & Leonhartsberger et al. (2001) \\
\hline GlnLG & $\begin{array}{l}\text { Glutamine/glutamate } \\
\text { level }\end{array}$ & Dual & $\begin{array}{l}\text { Dual (positive and } \\
\text { negative) }\end{array}$ & $\begin{array}{l}\text { FIS (positive) CRP } \\
\text { (dual) }\end{array}$ & $\begin{array}{l}\text { Inada et al. (1996), Nixon et al. (1986), Pahel et al. } \\
\text { (1982) }\end{array}$ \\
\hline \multicolumn{6}{|c|}{ Dependent systems } \\
\hline DcuRS & C4-dicarboxylates & Positive & No & $\begin{array}{l}\text { NarXL (negative) FNR } \\
\text { (positive) }\end{array}$ & Golby et al. (1999), Zientz et al. (1998) \\
\hline OmpREnvZ & Osmotic stress & Dual & No & $\begin{array}{l}\text { IHF (negative) CRP } \\
\text { (dual) }\end{array}$ & Mizuno et al. (1982), Nara et al. (1986) \\
\hline NarXL & Nitrate, nitrite & Dual & No & $\begin{array}{l}\text { ModE (positive) FNR } \\
\text { (negative) }\end{array}$ & Nohno et al. (1989), Stewart et al. (1989) \\
\hline RstAB & Magnesium & Dual & No & PhoPQ (positive) & Ogasawara et al. (2007) \\
\hline
\end{tabular}


a

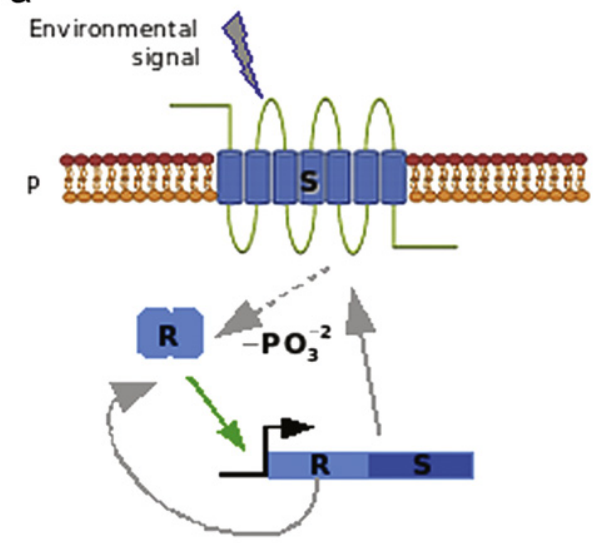

b

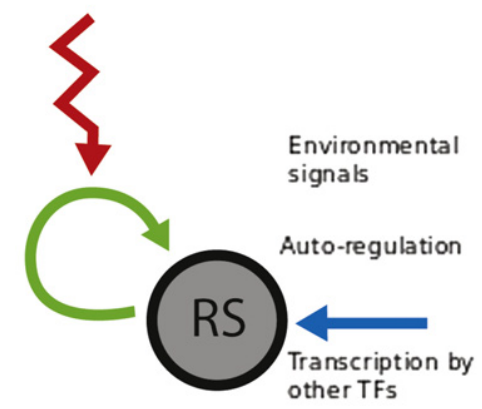

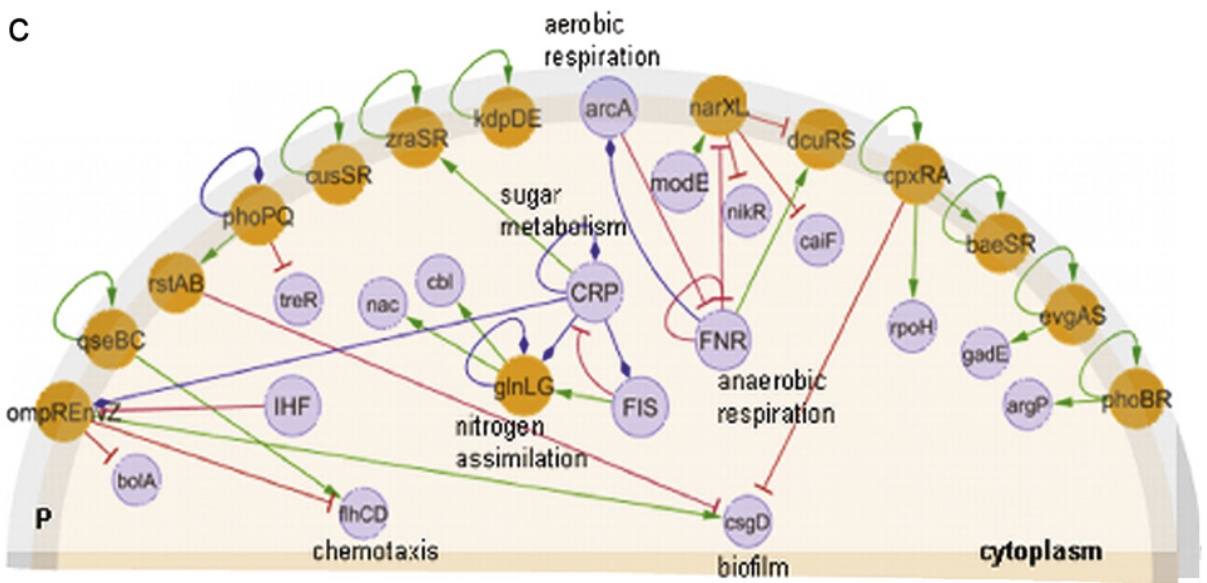

Fig. 1. Bacterial two-component systems: (a) Sketch of the genetic and protein components for sensing and transduction of the environmental information: $\mathrm{R}$ and $\mathrm{S}$ stand for regulator and sensor genes (and their proteins) respectively, solid grey arrows represent protein production, discontinuous grey arrow represents the phosphotransfer $\left(-\mathrm{PO}_{3}^{-2}\right)$ reaction from the sensor to the regulator, green arrow represents transcriptional activation. (b) Graphical representation of the transcriptional and environmental inputs. Node RS represents a two-component system, where each pair of genes encodes each of the 14 two-component systems contained in a single transcriptional unit (see text): the red arrow represents the input of the cognate signal, the green loop represents transcriptional auto-regulation, and the blue arrow represents the transcriptional inputs by additional regulators of the network. (c) Sub-network formed by the two-component systems and their first regulator neighbors (excluding noregulatory target genes) in E. coli. The two-component systems analyzed here are shown as orange nodes: red, green and blue lines and loops stand for repression, activation and dual (repression and activation) transcriptional activity respectively; P stands for periplasm. Some regulated physiological functions are shown.

nodes represent transcription factors executing regulatory decisions over their target genes (including themselves and other regulators). First we consider that each sensory system can be represented and modeled as a single node (Fig. 1b). This modeling simplification is supported by the following two considerations: each pair of genes encoding each of the 14 two-component systems is contained in a single transcriptional unit, and its component parts are expressed together and function as a single functional unit. Furthermore, we do not need to assume that regulator and sensor protein concentrations are the same, as long as the ratio of these concentrations remains constant. In this way we can model scenarios based on certain reports, where the protein abundance for the regulator is much higher than that for the sensor. Indeed, current experimental data reveal that only DcuR has an additional promoter not included in DcuS. Second, since nodes can be auto-regulated or not (previous section and Table 1), this auto-regulatory property is represented by loops on the nodes. Third, for functional switching, the sensor proteins should have specific signaling inputs, which we represent by a zigzag arrow over the loops on the nodes (Fig. 1b). Finally, nodes of semiautonomous and dependent groups have additional transcriptional inputs since other TFs can activate or repress them. These transcriptional inputs are represented by straight arrows ending on the nodes (Fig. 1b).

\subsubsection{Mathematical modeling}

We model the dynamic behavior of these sensory modules as a discrete-time dynamical system, open to the influence of external inputs. These models were introduced and studied in (Coutinho et al., 2006; Lima and Ugalde, 2006; Volchenkov and Lima, 2005), as a rigorously tractable alternative to the systems of piecewise affine differential equations (de Jong, 2002), or logical networks (Glass and Kauffman, 1973; Thomas et al., 1995), which are more commonly used to model regulatory networks.

In the specific case of two-component systems, we represent the state of a module at a given time by two numerical quantities:

(i) The equivalent (or proportional) production of the sensor and response proteins denoted by $x$ (Eqs. (1-7)).

(ii) The quantity of phosphorylated proteins denoted by $y$ (Eq. (9)).

Next we give details of both quantities $x$ and $y$. Later we explain the elements involved in the dynamic equations of $x$ corresponding to each type of system in our classification, and finally, we write the equation that describes the behavior of $y$. 
$x$ and $y$, take values in the interval between 0 and 1 , where 0 denotes the absence of protein and 1 its maximum level. In all cases (Figs. 2-4), we show the comparison between the total quantity protein $x$, and the fraction of phosphorylated protein, $y$. In our model, the time progression of each of these two quantities is governed by an equation allowing to compute $x$ and $y$ at time $t+1$ from: (a) its state at time $t$; (b) the action of other transcription factors modeled as external parameters in the equations, which can be positive $[P]$, negative $[N]$ or dual $[D]$, and can take the values 0 or 1 , depending on whether they are acting at a given time or not (explained below), (c) from the action of the cognate signal $\theta$, which also takes the values 1 or 0 depending on whether the signal is present or not respectively.

The equations also include degradation rates, denoted by $a$ and $b$, corresponding to dephosphorylated and phosphorylated proteins respectively. Each degradation rate takes a constant value between 0 and 1 .

The equations are normalized so that the value of the expression remains in the interval $[0,1]$ during the time course. Iterations of the dynamic equations correspond to an increase of the time by a unit whose physical value should be fixed from the experimental data.

Now we give generalities about $x$. In the literature, two types of regulation are usually considered, positive and negative, which are modeled by sigmoid curves (de Jong 2002, de Jong and Lima 2005). In the strongly non-linear regime, these curves can be approximated by discontinuous step functions. We use the Heaviside function

$H(x)= \begin{cases}0 & \text { if } x \leq 0 \\ 1 & \text { if } x>0\end{cases}$

to model this behavior. Since activation and inhibition thresholds are very close to 0 , we will use $H(x)$ and $1-H(x)$ for activation and inhibition respectively, and we will model the dual auto-regulation with the function $H(x-T)$, with $T$ different from 0 . These step functions model the way in which the system affects its own progression in the auto-regulatory loops. In the dual case the system first activates and then, when protein concentration reaches the value $T$, inhibits its own expression, which is equivalent to consider two different thresholds, 0 and $T$.

When several additional TFs are acting we introduce a parameter $k$, which indicates the relative strength of each these factors acting over a given two-component system.

Before we describe the model for each one of the classes in Table 1, let us explain the effect of regulation by additional TFs, which can be positive $[P]$, negative $[N]$ or dual $[D]$. Positive regulation implies that the product of other genes stimulates the rate of transcription $(x)$ of the two-component system, resulting in an increase of $x$. On the contrary, negative regulation implies that the product of that gene inhibits the transcription of the respective two-component system, i.e., $x$ decreases under the action of $N$. Finally, a dual regulator alternatively increases and reduces the transcription of a two-component system. Similarly to what happens in auto-regulation, regulation by other TFs can be modeled by Heaviside function on continuous variables, however, since we are only interested in the two-component system, it is enough for us to model the input of other TFs in terms of 1's for effective activation, and 0's for effective inhibition. The effect in the equations is coded as follows: positive regulation is represented by the letter $P$, and therefore $P=1$ most of the time; $N$ stands for negative regulation, hence $N=0$ most of the time; and $D$, representing a dual regulation, oscillates periodically taking values 0 and 1 .

Below we present the dynamic equations for $x$ according to the sub-classification presented above, and considering that for both, semiautonomous and dependent systems, only the existing configurations reported in the literature are analyzed (appearing in Table 1).

\subsubsection{Autonomous systems}

(1) Positive auto-regulation

$x^{t+1}=a x^{t}+(1-a)[s+(1-s) \theta]$.

Here the presence of the cognate signal, i.e. $\theta=1$, switches the equation from $x^{t+1}=a x^{t}+(1-a) s$ to $x^{t+1}=a x^{t}+(1-a)[s+(1-s)]$.

These systems require some baseline expression, which is associated with the term $s$ in the equation and ensures that $x^{t}>0$ for all time $t$. In the absence of the signal, i.e, when $\theta=0$ for a long period of time, $x$ approaches its baseline expression, and only under a persistent input $\theta=1$, the values of $x$ and $y$ reach their maximum level.

(2) Dual auto-regulation

$x^{t+1}=a x^{t}+(1-a)\left[s+(1-s) H\left(T-x^{t}\right) \theta\right]$.

This case is similar to the previous one; the presence of the signal changes the equation from $x^{t+1}=a x^{t}+(1-a) s$ to $x^{t+1}=a x^{t}+$ $(1-a)\left[s+(1-s) H\left(T-x^{t}\right)\right]$.

In absence of the cognate signal, the system behaves exactly as in the previous case. When, on the contrary, $\theta=1$, the existence of the threshold $T$ produces oscillations in the value of $x$.

\subsubsection{Semiautonomous systems}

(1) Positive auto-regulation

$x^{t+1}=a x^{t}+(1-a)\left[\theta+(1-\theta) P^{t}\right]$.

Here again, the effect of $\theta$ is to switch between two expressions of the dynamic equation of $x$. In this case the value of $\theta$ determines whether $x$ responds only to the autogenous regulation or only to the regulation by a positive regulator $P$.

(2) Dual auto-regulation

$x^{t+1}=a x^{t}+(1-a)\left[\theta H\left(T-x^{t}\right)+(1-\theta)\left(k D^{t}+(1-k) P^{t}\right)\right]$.

As in the cases above, $\theta$ indicates whether $x$ responds only to the auto-regulation or to regulation by other TFs. Here the autoregulation is dual and depends on a threshold $T$, which produces oscillations in the value of $x$. The system is regulated by two external TFs, one positive $[P]$ and the other dual $[D]$. The strength of the dual regulation relative to the positive one is given by a factor $k$ between 0 and 1 .

\subsubsection{Dependent systems}

(1) Regulation by a positive $\mathrm{TF}$

$x^{t+1}=a x^{t}+(1-a) P^{t}$

Here, and for each kind of dependent system described below, the presence of the cognate signal does not affect the dynamics of the sensor and response proteins, jointly represented by the variable $x$, but its effect can be observed in the amount of phosphorylated protein $y$. The value of $x$ in these systems is completely determined by the transcription via different TFs, in this case by a positive regulator $P$.

(2) Simultaneous regulation by a positive and a negative TF

$x^{t+1}=a x^{t}+(1-a)\left[k N^{t}+(1-k) P^{t}\right]$.

Here the value of $x$ responds only to the regulation by two external TFs, a negative $[N]$ and a positive $[P]$ one, and it is not affected by the cognate signal. The parameter $k$ indicates the relative strength of the negative regulation with respect to the positive one. 


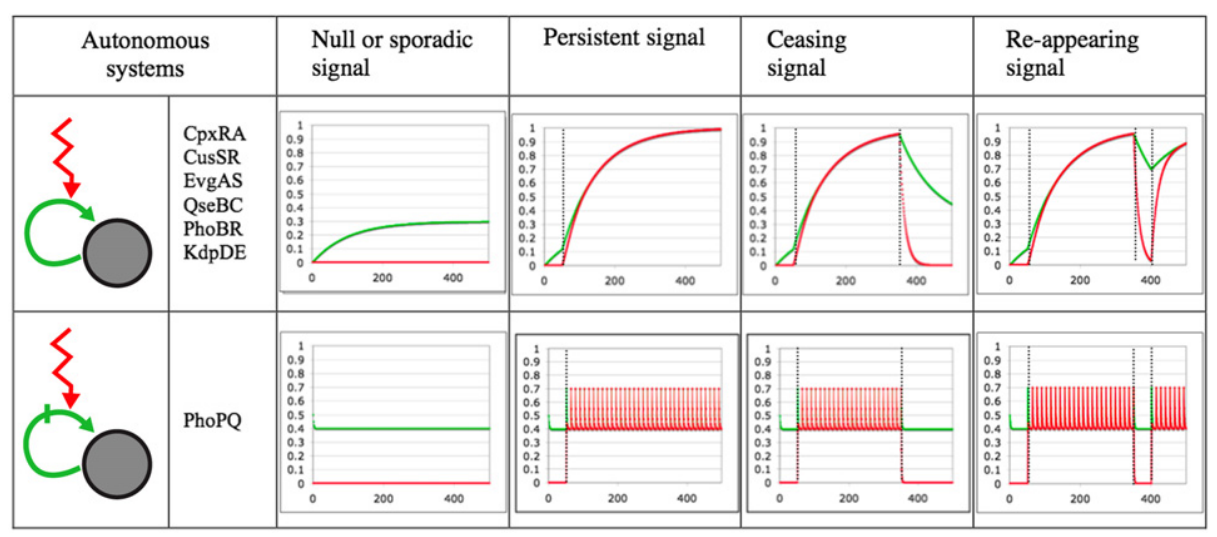

Fig. 2. Dynamics of autonomous two-component systems. Positive and dual self-regulations are shown in the first and second rows respectively.

In Figs. 2-4 the columns represent the behavior of the systems in response to the input signals. We consider the following cases for the signal: null or sporadic; persistent; ceasing; and re-appearing. The vertical dotted-lines indicate changes in the cognate signal. The "memory or inertial effect" appears clearly after the last signal change in the case of a reappearing signal. The green lines represent the total protein produced as a result of auto-regulation, and the red lines represent the total phosphorylated protein. In all figures we show 500 iterations of the map generating the dynamics.

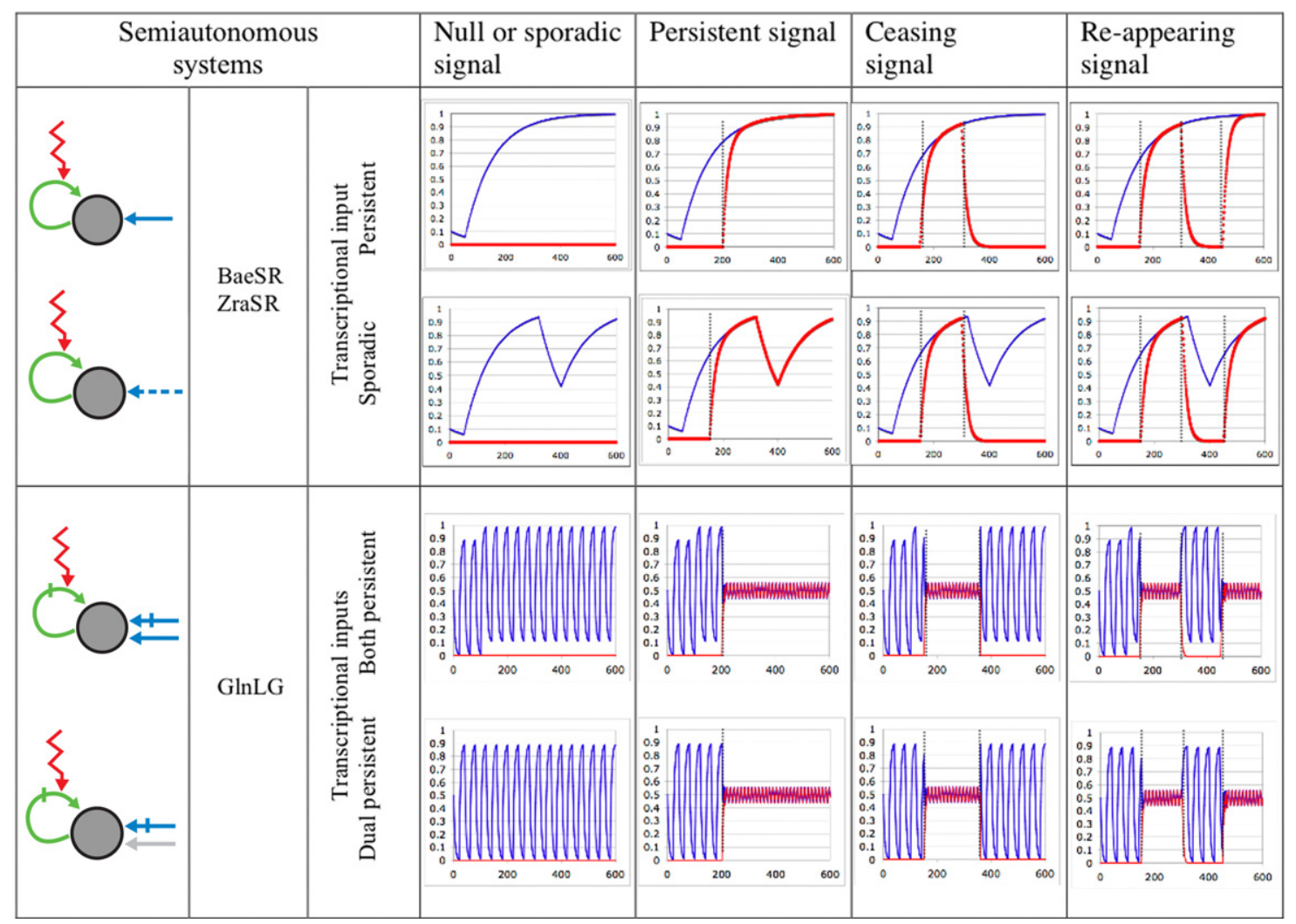

Fig. 3. Dynamics of semiautonomous two-component systems. The blue lines indicate the concentration of protein produced by the two-component system, as a result of the transcription by other TFs and by autoregulation. The first part of the figure shows the systems when they are positively regulated by other TFs and by themselves (BaeSR and ZraSR). The second part of the figure shows the systems when they are dually regulated by themselves and positive and dually regulated by other TFs (GlnLG). The first row shows the dynamics of the system when both TFs are acting, while the second row shows the dynamics when only a dual TF is acting. The presence of the cognate signal notoriously changes the frequency and amplitude in the oscillations. In all cases the red lines represent the total phosphorylated protein.

(3) Regulation by negative and dual regulators

$x^{t+1}=a x^{t}+(1-a)\left[k N^{t}+(1-k) D^{t}\right]$.

The dynamic behavior of $x$ is completely determined by the activity of both, a Negative and a dual regulator. The parameter $k$ determines which of them exerts the major influence. Dual regulation produces oscillations and negative regulation modulates the interval of these oscillations.
As already mentioned, the action of the cognate signal is responsible for the phosphorylation of the protein. The relative amount of phosphorylated protein, $\alpha=y / x$ depends on the persistence of the cognate signal, so that under the permanent presence of this signal, all the protein would be phosphorylated. We model this with the following formula

$\alpha^{t+1}=b \alpha^{t}+(1-b) \theta$, 


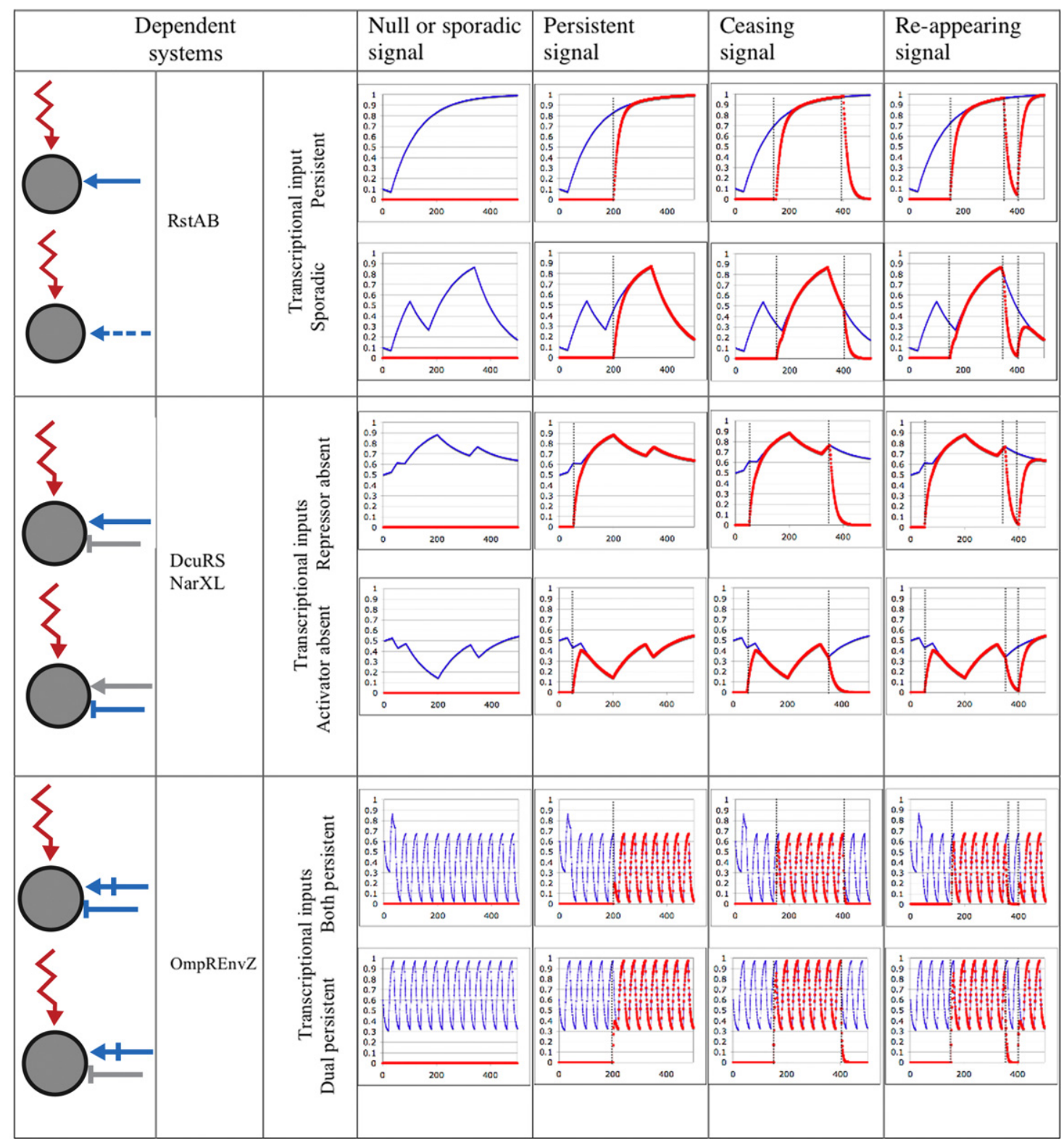

Fig. 4. Dynamics of dependent two-component systems. System depending on an activator (RstAB): the first row shows the system when the TF-input is persistent, and in the second we show a case in which this input is sporadic. Systems depending on an activator and a repressor (NarXL and DcuRS): The first row shows the behavior of the system when the activator is present and the repressor absent; the contrary is shown in the second row. Systems depending on a dual and repressor regulators (OmpR/ EnvZ): In the first and second rows we show the behavior in the presence and absence of the repressor, the dual regulator is assumed to be present in both conditions. In all cases, the blue lines indicate the total concentration of the protein produced by the system, while the red lines represent the amount of phosphorylated protein.

where $\alpha^{t+1}$ takes values between 0 and 1 , and represents the fraction of the protein that has been phosphorylated at time $t+1$ as a function of the same fraction at time $t$. The parameter $b$ denotes the dephosphorylation rate of phosphorylated protein, then, if the value of $\theta$ is constantly 0 , the fraction of phosphorylated protein tends to 0 .

Finally, the amount of phosphorylated protein at time $t$ is given by the expression:

$y^{t}=x^{t} \alpha^{t}$

Let us remember that the state of a module at time $t$ is represented by two numerical quantities: the quantity of phosphorylated proteins denoted by $y^{t}$, and the equivalent (or proportional) production of the sensor and response proteins denoted by $x^{t}$. Alternatively we could use $x^{t}$ and $\alpha^{t}$ to present the state of the system.

If the production of the sensor and regulator proteins is not equivalent but proportional, then the total amount of these proteins would be determined from $x^{t}$ with the help of two proportionality constants to be determined from experimental data. Since we are not interested in total amounts but in relative concentrations, the sole variables $x$ and $y$, or $x$ and $\alpha$, are sufficient to completely describe the state of the system.

The values of the parameters used in the simulations were fixed to qualitatively reproduce the behavior observed in known situations. For instance, the degradation rates $a$ and $b$ of the non-phosphorylated and phosphorylated protein respectively were fixed so that the process of activation is slower than inhibition. To determine $k$, we keep in mind the influence on transcription of the additional TFs. 
In the analysis of the behavior of the two-component systems we distinguish four dynamic states: the resting state, when the system has a minimum or null level of phosphorylated proteins; induction of the system, when there is a noticeable increase in the level of phosphorylated proteins from this minimum level; the turn off of the system, when the level of expression drops and eventually returns to the resting state; and the multistationarity of the system, when we observe (monotonous) transitions between potential steady states, or between different possible oscillatory modes.

Below we examine the dynamic behavior of each kind of two-component system in response to the input signals. We consider the following cases for the signal: null or sporadic, if $\theta=0$ most of the time, and seldom equals 1 ; persistent, if $\theta=1$ most of the time and only occasionally equals 0 (in Figs. 2-4 we consider the case $\theta=0$ for a short period and 1 for the rest of the time); ceasing, when $\theta=0$ in a short initial time, 1 in a subsequent interval and 0 for the rest of the time; and re-appearing, when it follows the previous sequence of $\theta$ 's but finally stabilizes at 1 .

\subsection{Dynamic behavior of two-component systems}

\subsubsection{Autonomous systems}

In these two-component systems we find positive and dual self-regulation (first and second rows in Fig. 2). Autonomous systems necessarily require some baseline expression even in the absence of their cognate signal. This baseline expression derives from a transcriptional escape or, in some cases, is due to a constitutive promoter in addition to the auto-regulated one, as in PhoPQ (Miyashiro and Goulian, 2007). If on the contrary, the system reaches a null transcription level, the absence of the sensor protein would make the system blind to the cognate signal when it is present.

The induction of these systems takes place only if the cognate signals are strong and persistent enough. Since the molecular process of protein production is slow and the weak promoters of activated genes turn out to be more efficient as more phosphorylated activators are present, the time-response of positive circuits is slow, and delayed with respect to negative or dual circuits (Alon, 2007). In addition, the sluggish accumulation of phosphorylated proteins requires a persistent signal in order to pass a threshold above which the systems are robust for input/output signaling.

The mechanisms for the turn off of these systems depend on their regulatory mode. In the case of positive self-regulation, if the signal is persistent, the protein increases up to a maximum level of expression; the degradation of the protein is compensated by its continuous production, and it only decreases at each cell division. On the contrary, if the signal disappears, most of sensor proteins could directly cause the dephosphorylation of the response regulators resulting in their inactivation. Nonetheless, since the proteins are not immediately degraded, and some pairs have been found forming protein complexes, it is possible that these complexes could persist for some time. In this situation, if the signal appears again the transcriptional response should be faster compared to the first time the signal appeared. This is due to the presence of accumulated regulators ready to act as soon as they are again phosphorylated by the sensor. We interpret this rapid (learned) response to a known stimulus as a "memory or inertial effect" (resembling the immune system). Note that this phenomenon of "memory or inertia" might be present in all the systems analyzed here, although the response could only be selfsustained and amplified by the autonomous systems.

As a general rule, circuits with positive auto-regulation are bistable (Ajo-Franklin et al., 2007; Thomas, 1998). In E. coli the following two-component systems are positively auto-regulated: CpxRA, CusSR, EvgAS, QseBC, PhoBR and KdpDE (Table 1). As expected, we observe a bistable behavior for both the nonphosphorylated and phosphorylated proteins.

On the other hand, PhoPQ behaves as a dual auto-regulator. This means PhoP could regulate itself both positively and negatively, depending on the presence of its effector signal. The dual auto-regulation implies that the system never falls to zero, but oscillates as a homeostatic controller, between a basal and a maximal level.

\subsubsection{Semiautonomous systems}

This group exhibits a more complex behavior. This complexity is due to their auto-regulation, and to their regulation by other TFs found in the transcriptional machinery of the bacterium (Fig. 1c). Since autonomous systems were described above, here we will focus our discussion on the conditions where the systems are modulated by additional regulators.

In this subclass of two-component systems we find two cases of regulation: positive regulation by other TFs and positive autoregulation (BaeSR and ZraSR); and regulation by two other TFs one of which provides dual-regulation and the other provides positive-regulation, in addition to dual auto-regulation (GlnLG) (first and second rows in Fig. 3). Semiautonomous systems can work by indirectly integrating information (sensed by the TFs regulating them) in the absence of their cognate signal. In this condition, one prediction is that the persistent action of the other TFs allows the system to reach its maximal level of expression with the dual regulator producing oscillations between a basal and a maximal level.

Turning on the systems: It could be that the quantity of protein depends completely on the activity of the additional regulators; however the cognate signals should be strong and persistent enough to permit the system to maintain its autonomy.

Turning off the system: when the signal disappears, the sensor protein may cause the dephosphorylation of the response protein, although this does not affect the degradation rate of the twocomponent's product.

As for the autonomous systems, we observe here an "inertial effect". If the signal appears again, the transcriptional response related to the quantity of phosphorylated protein should be faster compared to the first time it appears.

Changes in the concentration of the phosphorylated proteins, for the semiautonomous systems, have the same effect as in the autonomous ones; for instance, the phosphorylated proteins in systems with positive auto-regulation, BaeSR and ZraSR, have a bi-stable behavior. On the other hand, GlnLG behaves as a dual auto-regulator, oscillating between basal and maximal levels.

\subsubsection{Dependent systems}

In this subclass we find three possible cases of regulation by other TFs: positive (RstAB); positive and negative (DcuRS and NarXL); and dual-regulation in combination with negative regulation (OmpREnvZ) (first, second, and third rows in Fig. 4). The transcription of these systems depends exclusively on the activity of the other TFs of the regulatory network. As a consequence, the first and second cases can be totally turned off or reach their maximum levels of expression in absence of the signal. The third case is always oscillating depending on the CRP's own oscillation.

Thus the induction of the first two cases only takes place if their regulators are active. Once these dependent systems are turned on, they might act without their signals or require the persistency of their cognate signal to regulate their target genes; e.g. activating in one conformation and repressing in the other. 
This may be the case since we observe that most of them have dual activity on their target genes (third column on Table 1). In this way, these systems could be considered as intermediate in regulatory pathways. Again, if their signals disappear, the sensor protein could directly cause the dephosphorylation of the response regulator resulting in their inactivation or a change in their active conformation. This dephosphorylation does not imply, as for the semiautonomous systems, that the quantity of protein decreases. Then if the signal appears again, these systems also present the "inertial effect" as in the two previous cases.

NarXL is activated and repressed by ModE and FNR respectively; therefore it could exhibit several steady states under the persistent action of either or both of these two regulators. Changes in the action of these regulators lead the system from one possible steady state to another one. On the other hand, OmpREnvZ presents a homeostatic behavior, in accordance with CRP oscillations, even when it is repressed by the additional action of IHF. This system might also present several frequencies of oscillation. The persistency of the dependent systems completely relies on the activity of their regulating TFs.

\section{Discussion}

Bacterial two-component sensory systems are, in general, controlling social interactions with other organisms and responding to multiple environmental stress conditions by sensing molecules of quorum-sensing, ions, drugs and physicochemical stresses on membrane proteins, etc. Here we analyzed 14 experimentally characterized two-component systems in E. coli and showed how these signal-transduction modules might perform in bacteria. Two-component autonomous systems could be considered as the devices for controlling the input of exogenous information into the cell physiology, constituting "origons", or informational entries in the transcriptional regulatory network (Balazsi et al., 2005). Thus these sensory systems, although not regulated by additional TFs can themselves regulate other TFs and more importantly, co-regulate with them and in this way integrate information about exogenous conditions with the endogenous conditions perceived by the rest of the regulatory network (Fig. 1c and 5).

The activity of semiautonomous and dependent systems relies on the integration of external and endogenous conditions, since most of the TFs regulating them use small-molecule metabolites produced endogenously as effectors. The interactions of the semiautonomous systems with the rest of the regulatory network are as follows: BaeSR connects its activity to that of CpxR (of the autonomous group), which results in a robust device as two positive circuits are coupled, while ZraSR connects its activity to CRP (Fig. 1c). GlnLG might be considered as a super-homeostatic controller since in addition to itself, it is dually regulated by CRP (Table 1). Thus, the GlnLG system might be oscillating on at least two frequencies. Moreover, it might be that GlnLG tends to be more active than repressed because of its additional activation by FIS. Finally, given that CRP dually regulates FIS and FIS represses CRP, they constitute negative or positive feedback circuits depending on the way CRP regulates FIS (Fig. 1c). All these controls reflect the importance of GlnLG as one of the few twocomponent systems responding to endogenous conditions, in this case by regulating nitrogen metabolism.

Looking at the transcription of the dependent systems it is possible to find a complex connection with the rest of the transcriptional network. For instance, NarXL is activated and repressed by ModE and FNR respectively; on the other hand, NarXL represses DcuRS. Furthermore, DcuRS is also activated by

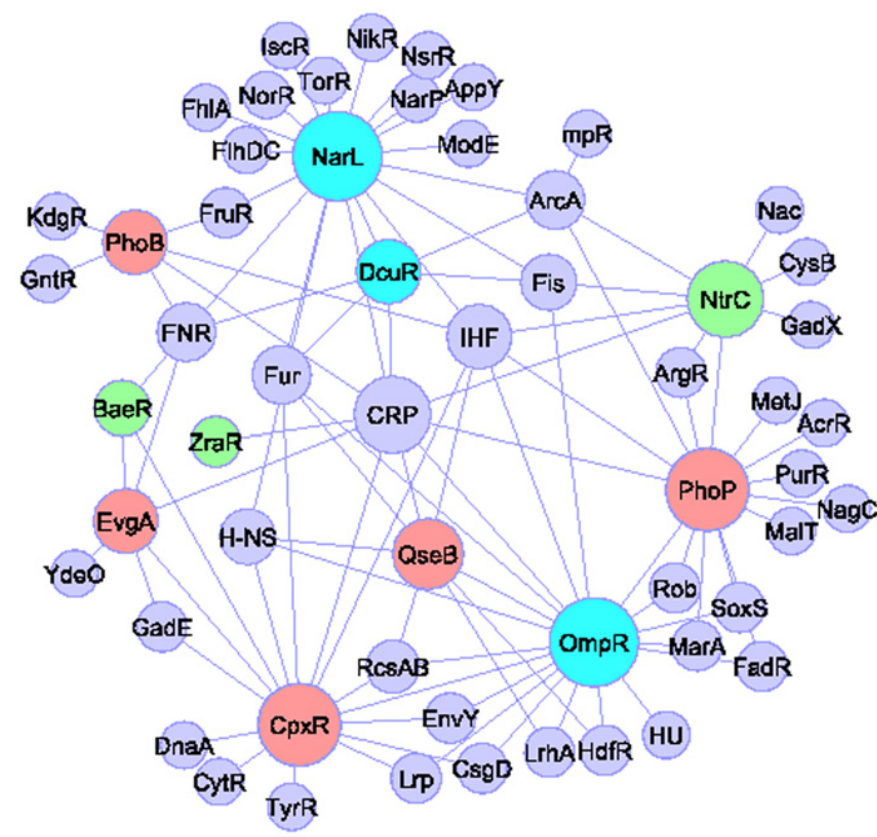

Fig. 5. Co-regulatory activity, on known promoters, of two-component systems with other TFs of the regulatory network in E. coli. Pink, green and light-blue nodes represent TFs of autonomous, semiautonomous and dependent two-component systems respectively. Node sizes represent their degree of co-regulation (connectivity) with other TFs without considering the number of promoters which they co-regulate. (For interpretation of the references to the color in this figure legend, the reader is referred to the web version of this article.)

FNR. In this way FNR, NarXL and DcuRS constitute a coherent feed-forward loop network motif (the product of the interactions is positive) (Mangan et al., 2003). This implies that the full activation of DcuRS depends primarily on the activity of FNR. This last example implies a complex informational processing of external and internal signals in order to control anaerobic respiration in E. coli (Fig. 1c).

The mechanism for dual auto-regulation could be explained if TFs bind differentially to strong and weak DNA-binding sites within the same regulatory region; e.g. in their non-phosphorylated state the TFs could bind only to strong sites (and activate) while in their phosphorylated form the TFs also bind to weak sites, occupying more sites on the DNA and resulting in the interference of RNA polymerase activity (repressing). Experimental validation of our models is pending. For that purpose, one can choose a system in each group, construct transcriptional fusions with fluorescent proteins and follow their expression under relevant conditions at different time points and in mutant regulator strains as we are currently doing with the GlnLG sensory system.

The performance of most two-component systems as positive switches might explain the phenotypic heterogeneity observed in bacterial populations with a common genetic background (Karmakar and Bose, 2007; Maamar et al., 2007). This heterogeneity might be explained by noisy background signals causing some of these multistationary systems to operate in one of their steady states, especially if we consider that bacteria may be distributed throughout the local environment and exposed to different gradients of environmental signals. As result, different sets of genes might be expressed in individuals or colonies at different locations within the population. Furthermore, in a previous study it was found that two-component systems are located on the periphery of the regulatory network and the activity of some of them might be to define the last steps for biofilm and flagella 
formation (Martinez-Antonio et al., 2008). Furthermore, it has been found experimentally that these developmental processes give rise to a mixture of phenotypes in bacterial populations (Sauer et al., 2002).

This study could pave the way for the design of sensory devices or regulatory circuits for coupling exogenous and endogenous sensing modules, or to produce a specific cellular response. On the other hand, in this study our aim is to model the most common properties of standard two-component systems. A direction of future research is to study in detail the specific characteristics of each system, since in some of them additional components could serve as intermediates in the signaling pathway: these intermediates seem to function as a means for extra control of the system, rather than a signal amplification trigger (Gao and Stock, 2009; Mitrophanov and Groisman, 2008). There are also possibilities of cross-talk between these systems; however this does not seem to be the rule, as most of them tend to be specific in their phosphotransfer reactions (Gao and Stock, 2009; Groban et al., 2009), and these specificities increase with the concentration of protein as a result of auto-regulation. Thus in the presence of a persistent signal the activity of the systems is clearest and robust. Finally, it also remains to be understood how the variant genomes and their regulatory networks are affected by the information provided by the environment, i.e., how the information defining the relevant environmental variables leaves its hallmark on the genomes and how this "memory or inertial effect" is stored in the network of interactions of the adapted bacteria.

\section{Materials and methods}

\subsection{Biological data}

Biochemical data of two-component systems from $E$. coli were recovered from Ecocyc (Keseler et al., 2005) and the transcriptional information on their signal effectors and transcriptional regulation from Regulon DB (Salgado et al., 2006). The regulatory interactions were drawn with the Cytoscape program (Shannon et al., 2003).

\section{Acknowledgments}

We thank fruitful discussions with June Simpson, Amanda Castillo, Enrique Balleza, Gelasio Salazar and Alex De Luna. We also thank Ana L. Hernandez and Shareny V. Cabrera Zago for their assistance with the figures. This work was partially supported by: CONACyT (Grant 48352-E), and by SEP through its PROMEP program given to E.U.; CONCYTEG (young researcher) given to A.M-A.; and by Sofia Kovalévskaia and CONACyT Ph.D. fellowships granted to B.L. We thank the CIC A.C. in Cuernavaca for the facilities offered to us during the meeting "Dynamics of Regulatory Networks II" held in summer 2007.

\section{References}

Ajo-Franklin, C.M., Drubin, D.A., Eskin, J.A., Gee, E.P. Landgraf, D., Phillips, I., Silver, P.A., 2007. Rational design of memory in eukaryotic cells. Genes Dev. 21, 2271-2276.

Alon, U., 2007. An Introduction to Systems Biology: Design Principles of Biological Circuits. Chapman \& Hall/CRC, Boca Raton, FL.

Asha, H., Gowrishankar, J., 1993. Regulation of kdp operon expression in Escherichia coli: evidence against turgor as signal for transcriptional control. J. Bacteriol. 175, 4528-4537.

Balazsi, G., Barabasi, A.L., Oltvai, Z.N., 2005. Topological units of environmenta signal processing in the transcriptional regulatory network of Escherichia coli. Proc. Natl. Acad Sci. USA 102, 7841-7846.
Blattner, F.R., Plunkett 3rd, G., Bloch, C.A., Perna, N.T., Burland, V., Riley, M., Collado-Vides, J., Glasner, J.D., Rode, C.K., Mayhew, G.F., Gregor, J., Davis, N.W., Kirkpatrick, H.A. Goeden, M.A., Rose, D., Mau, B., Shao, Y, 1997. The complete genome sequence of Escherichia coli K-12. Science 277, 1453-1474.

Coutinho, R., Fernandez, B., Lima, R., Meyroneinc, A., 2006. Discrete time piecewise affine models of genetic regulatory networks. J. Math. Biol. 52, 524-570.

de Jong, H., 2002. Modeling and simulation of genetic regulatory systems: a literature review. J. Comput. Biol. 9, 67-103.

de Jong, H., Lima, R., 2005. Modeling the dynamics of genetic regulatory networks: continuous and discrete models. Lect. Notes Phys. 671, 307-340.

DiGiuseppe, P.A., Silhavy, T.J., 2003. Signal detection and target gene induction by the CpxRA two-component system. J. Bacteriol. 185, 2432-2440.

Gao, R., Stock, A.M., 2009. Biological insights from structures of two-component proteins. Annu. Rev. Microbiol. 63, 133-154.

Glass, L., Kauffman, S.A., 1973. The logical analysis of continuous, non-linear biochemical control networks. J. Theor. Biol. 39, 103-129.

Golby, P., Davies, S., Kelly, D.J., Guest, J.R., Andrews, S.C., 1999. Identification and characterization of a two-component sensor-kinase and response-regulator system (DcuS-DcuR) controlling gene expression in response to C4-dicarboxylates in Escherichia coli. J. Bacteriol. 181, 1238-1248.

Groban, E.S., Clarke, E.J., Salis, H.M., Miller, S.M., Voigt, C.A., 2009. Kinetic buffering of cross talk between bacterial two-component sensors. J. Mol. Biol. 390 380-393.

Guespin-Michel, J., Kaufman, M., 2001. Positive feedback circuits and adaptive regulations in bacteria. Acta Biotheor. 49, 207-218.

Guhaniyogi, J., Wu, T., Patel, S.S., Stock, A.M., 2008. Interaction of CheY with the Cterminal peptide of CheZ. J. Bacteriol. 190, 1419-1428.

Hirakawa, H., Inazumi, Y., Masaki, T., Hirata, T., Yamaguchi, A., 2005. Indole induces the expression of multidrug exporter genes in Escherichia coli. Mol. Microbiol. 55, 1113-1126.

Igo, M.M., Slauch, J.M., Silhavy, T.J., 1990. Signal transduction in bacteria: kinases that control gene expression. New Biol. 2, 5-9.

Inada, T., Takahashi, H., Mizuno, T., Aiba, H., 1996. Down regulation of cAMP production by cAMP receptor protein in Escherichia coli: an assessment of the contributions of transcriptional and posttranscriptional control of adenylate cyclase. Mol. Gen. Genet. 253, 198-204.

Karmakar, R., Bose, I., 2007. Positive feedback, stochasticity and genetic competence. Phys. Biol. 4, 29-37.

Kasahara, M., Nakata, A., Shinagawa, H., 1992. Molecular analysis of the Escherichia coli phoP-phoQ operon. J. Bacteriol. 174, 492-498.

Kato, A., Tanabe, H., Utsumi, R., 1999. Molecular characterization of the PhoP-PhoQ two-component system in Escherichia coli K-12: identification of extracellular Mg2+-responsive promoters. J. Bacteriol. 181, 5516-5520.

Keseler, I.M., Collado-Vides, J., Gama-Castro, S., Ingraham, J., Paley, S., Paulsen, I.T., Peralta-Gil, M., Karp, P.D., 2005. EcoCyc: a comprehensive database resource for Escherichia coli. Nucleic Acids Res. 33 (Database Issue), D334-D337.

Leonhartsberger, S., Huber, A., Lottspeich, F., Bock, A., 2001. The hydH/G Genes from Escherichia coli code for a zinc and lead responsive two-component regulatory system. J. Mol. Biol. 307, 93-105.

Lima, R., Ugalde, E., 2006. Dynamical complexity of discrete-time regulatory networks. Nonlinearity 19, 237-259.

Maamar, H., Raj, A., Dubnau, D., 2007. Noise in gene expression determines cell fate in Bacillus subtilis. Science 317, 526-529.

Mangan, S., Zaslaver, A., Alon, U., 2003. The coherent feedforward loop serves as a sign-sensitive delay element in transcription networks. J. Mol. Biol. 334 197-204.

Martinez-Antonio, A., Janga, S.C., Thieffry, D., 2008. Functional organisation of Escherichia coli transcriptional regulatory network. J. Mol. Biol. 381, 238-247.

Martinez-Antonio, A., Janga, S.C., Salgado, H., Collado-Vides, J., 2006. Internalsensing machinery directs the activity of the regulatory network in Escherichia coli. Trends Microbiol. 14, 22-27.

Mascher, T., Helmann, J.D., Unden, G., 2006. Stimulus perception in bacterial signal-transducing histidine kinases. Microbiol. Mol. Biol. Rev. 70, 910-938.

Mitrophanov, A.Y., Groisman, E.A., 2008. Signal integration in bacterial twocomponent regulatory systems. Genes Dev. 22, 2601-2611.

Miyashiro, T., Goulian, M., 2007. Stimulus-dependent differential regulation in the Escherichia coli PhoQ PhoP system. Proc. Natl. Acad. Sci. USA 104, 16305-16310.

Mizuno, T., Wurtzel, E.T., Inouye, M., 1982. Cloning of the regulatory genes (ompR and envZ) for the matrix proteins of the Escherichia coli outer membrane. J. Bacteriol. 150, 1462-1466.

Munson, G.P., Lam, D.L., Outten, F.W., O'Halloran, T.V., 2000. Identification of a copper-responsive two-component system on the chromosome of Escherichia coli K-12. J. Bacteriol. 182, 5864-5871.

Nara, F., Mizuno, T., Mizushima, S., 1986. Complementation analysis of the wild-type and mutant ompR genes exhibiting different phenotypes of osmoregulation of the ompF and ompC genes of Escherichia coli. Mol. Gen. Genet. 205, 51-55.

Nishino, K., Honda, T., Yamaguchi, A., 2005. Genome-wide analyses of Escherichia coli gene expression responsive to the BaeSR two-component regulatory system. J. Bacteriol. 187, 1763-1772.

Nixon, B.T., Ronson, C.W., Ausubel, F.M., 1986. Two-component regulatory systems responsive to environmental stimuli share strongly conserved domains with the nitrogen assimilation regulatory genes ntrB and ntrC. Proc. Natl. Acad. Sci. USA 83, 7850-7854.

Nohno, T., Noji, S., Taniguchi, S., Saito, T., 1989. The narX and narL genes encoding the nitrate-sensing regulators of Escherichia coli are homologous to a family of 
prokaryotic two-component regulatory genes. Nucleic Acids Res. 17, 2947-2957.

Ogasawara, H., Hasegawa, A., Kanda, E., Miki, T., Yamamoto, K., Ishihama, A., 2007. Genomic SELEX search for target promoters under the control of the PhoQPRstBA signal relay cascade. J. Bacteriol. 189, 4791-4799.

Oshima, T., Aiba, H., Masuda, Y., Kanaya, S., Sugiura, M., Wanner, B.L., Mori, H., Mizuno, T., 2002. Transcriptome analysis of all two-component regulatory system mutants of Escherichia coli K-12. Mol. Microbiol. 46, 281-291.

Otto, K., Silhavy, T.J., 2002. Surface sensing and adhesion of Escherichia coli controlled by the Cpx-signaling pathway. Proc. Natl. Acad. Sci. USA 99, 2287-2292.

Pahel, G., Rothstein, D.M., Magasanik, B., 1982. Complex glnA-glnL-glnG operon of Escherichia coli. J. Bacteriol. 150, 202-213.

Salgado, H., Gama-Castro, S., Peralta-Gil, M., Diaz-Peredo, E., Sanchez-Solano, F., Santos-Zavaleta, A., Martinez-Flores, I., Jimenez-Jacinto, V., Bonavides-Martinez, C., Segura-Salazar, J., Martinez-Antonio, A., Collado-Vides, J., 2006. RegulonDB (version 5.0): Escherichia coli K-12 transcriptional regulatory network, operon organization, and growth conditions. Nucleic Acids Res. 34, D394-D397.

Sauer, K., Camper, A.K., Ehrlich, G.D., Costerton, J.W., Davies, D.G., 2002. Pseudomonas aeruginosa displays multiple phenotypes during development as a biofilm. J. Bacteriol. 184, 1140-1154.

Shannon, P., Markiel, A., Ozier, O., Baliga, N.S., Wang, J.T., Ramage, D., Amin, N., Schwikowski, B., Ideker, T., 2003. Cytoscape: a software environment for integrated models of biomolecular interaction networks. Genome Res. 13, $2498-2504$.

Sperandio, V., Torres, A.G., Kaper, J.B., 2002. Quorum sensing Escherichia coli regulators $B$ and $C$ (QseBC): a novel two-component regulatory system involved in the regulation of flagella and motility by quorum sensing in E. coli. Mol. Microbiol. 43, 809-821.

Stewart, V., Parales Jr., J., Merkel, S.M., 1989. Structure of genes narL and narX of the nar (nitrate reductase) locus in Escherichia coli K-12. J. Bacteriol. 171, 2229-2234.

Tanabe, H., Masuda, T., Yamasaki, K., Katoh, A., Yoshioka, S., Utsumi, R., 1998. Molecular interaction between proteins involved in EvgAS signal transduction of Escherichia coli. Biosci. Biotechnol. Biochem. 62, 78-82.
Thomas, R., 1973. Boolean formalization of genetic control circuits. J. Theor. Biol. $42,563-585$

Thomas, R., 1998. Laws for the dynamics of regulatory networks. Int. J. Dev. Biol. 42, 479-485.

Thomas, R., D’Ari, R., 1990. Biological Feedback. Boca Raton, CRC Press, Florida, USA. Thomas, R., Thieffry, D., Kaufman, M., 1995. Dynamical behaviour of biological regulatory networks-I. Biological role of feedback loops and practical use of the concept of the loop-characteristic state. Bull Math. Biol. 57, 247-276.

Volchenkov, D., Lima, R., 2005. Random shuffling of switching parameters in a model of gene expression regulatory network. Stochastics Dyn. 5, 75-95.

Walderhaug, M.O., Polarek, J.W., Voelkner, P., Daniel, J.M., Hesse, J.E., Altendorf, K., Epstein, W., 1992. KdpD and KdpE, proteins that control expression of the kdpABC operon, are members of the two-component sensor-effector class of regulators. J. Bacteriol. 174, 2152-2159.

Wanner, B.L., Chang, B.D., 1987. The phoBR operon in Escherichia coli K-12. J. Bacteriol. 169, 5569-5574.

Whitworth, D.E., Millard, A., Hodgson, D.A., Hawkins, P.F., 2008. Protein-protein interactions between two-component system transmitter and receiver domains of Myxococcus xanthus. Proteomics 8, 1839-1842.

Workentine, M.L., Chang, L., Ceri, H., Turner, R.J., 2009. The GacS-GacA twocomponent regulatory system of Pseudomonas fluorescens: a bacterial twohybrid analysis. FEMS Microbiol. Lett. 292, 50-56.

Yamamoto, K., Ishihama, A., 2005. Transcriptional response of Escherichia coli to external copper. Mol. Microbiol. 56, 215-227.

Zhao, X., Copeland, D.M., Soares, A.S., West, A.H., 2008. Crystal structure of a complex between the phosphorelay protein YPD1 and the response regulator domain of SLN1 bound to a phosphoryl analog. J. Mol. Biol. 375, 1141-1151.

Zientz, E., Bongaerts, J., Unden, G., 1998. Fumarate regulation of gene expression in Escherichia coli by the DcuSR (dcuSR genes) two-component regulatory system. J. Bacteriol. 180, 5421-5425.

Zoetendal, E.G., Smith, A.H., Sundset, M.A., Mackie, R.I., 2008. The BaeSR twocomponent regulatory system mediates resistance to condensed tannins in Escherichia coli. Appl. Environ. Microbiol. 74, 535-539. 